\title{
Fault Tolerant Cost-effective Carrierless Stochastic Synthesis of Voltages and Currents in Multi-Cell Multilevel Converters via the Central Limit Theorem
}

\author{
Pierluigi Tenca \\ IEEE Member (formerly at ABB Corporate Research) \\ Munich, D-80802, Germany \\ E-mail: infxxvi@tin.it
}

\author{
Luca Peretti \\ ABB Corporate Research \\ Västerås, 72178, Sweden \\ E-mail: luca.peretti@se.abb.com
}

\begin{abstract}
The paper presents - with experimental evidence a highly fault tolerant, cost-effective and carrierless solution to synthesize voltages (or currents) across the series (or parallel) connection of several voltage-source (or current-source) cells. Hundreds of voltage-source cells already characterize modern multilevel HVDC converters and structures based on currentsource cells could become industrially meaningful too, if sufficient advancements in semiconductor and magnetic materials will occur. Exactly by relying on a large number of cells, the key approach is characterized by an unconventional absence of any determinism. The voltages (or currents) of all cells are generated as discrete independent random variables whose distribution is parameterized by the desired reference waveform to be synthesized. This is a radical difference from the already existing random PWM modulations, where only the switching instants are aleatory. The essence of the proposed method lies in observing that multilevel converters, which synthesize the desired waveforms through additive linear combinations (e.g. the simplest sum) of several elementary contributions, become naturally ruled by the Central Limit Theorem of the theory of probablity. Such a fundamental law of nature assures also that the greater the number of cells, the more the waveform synthesis becomes inherently robust, fault-tolerant and accurate. This property strikingly differs from those of deterministic centrally ruled modulations and it is also achieved with reduced hardware complexity and cost. The proposed method benefits from emerging technologies employing smaller mass-produced cells based on devices capable of improved switching characteristics, like $\mathrm{SiC}$ or $\mathrm{GaN}$, thereby suiting design philosophies characterized by a high number of cost-optimized standard elementary units. Equally important, the proposed synthesis eliminates most physical communication channels (e.g. optical fibers) among the controller and the switches, thereby greatly improving the system reliability. The paper introduces also a hybrid variant of the method conceived for fewer cells.
\end{abstract}

\section{INTRODUCTION}

The presently most common approach for synthesizing voltage (or current) waveforms in high-power voltage-source (or current-source) multilevel converters, based on several elementary cells connected in series (or parallel), is still deterministic and it relies on a multiplicity of synchronized carriers, which can be easily by the hundreds nowadays. A well known example of such class of converters is the M2LC structure by Prof. R. Marquardt [1]-[4]. Such a methodology poses significant reliability requirements on the main controller, which must assure a significant level of availability in the difficult scenario characterized by a large number of signal lines to and from the controllable switches. These physical communication channels are easily by the hundreds as well nowadays, even when multiplexing techniques together with addressing schemes of the cells are used [5]. Still today, several optical fibers and their associated optoelectronic transmitters/receivers are the most common physical media for the switching signals, as well as for the feedback/acknowledge signals, to and from the controllable switches. Some realizations generate the switching signals inside the cells upon reception of the voltage command. Other realizations let the central controller to command directly each single switch. Whichever the realization, the present concepts for multilevel synthesis are intrinsically deterministic and the higher the number of cells the greater the complexity of the command chains - then their costs - become in order to assure the needed high system availability, which is so crucial for power system applications [6]. The envisaged future ratings of HVDC and DC-GRID converters can easily demand a so high number of cells, for which it will become increasingly expensive to guarantee a reliable "deterministic" control hardware capable of maintaining an acceptably low probability of critical malfunctions in the command chains. By eliminating such needs [4], [6] - some of which are consequences of approaching the waveform syntheses in a deterministic way - one can greatly improve the system availability and reduce the system life cycle costs. The paper describes a different and non-deterministic approach to the synthesis of multilevel waveforms in the aforementioned class of converters. Its principles were already disclosed in the publicly available document [7] by Research Disclosure. The approach owes its attractive characteristics to an unconventional absence of determinism. Its conceptual roots lie in the Central Limit Theorem (CLT) of the Theory of Probability [8], a fundamental law of nature ruling many phenomena that manifest additive effects in large multitudes of similar, yet independent, elementary physical entities. When several voltage-source (current-source) cells are in series (parallel), they constitute a physical system composed by many elementary entities indeed. If the interest is on the total voltage (current) at the two extreme terminals of the series (parallel), this becomes exactly a scenario where such a total voltage (current) can be ruled stochastically - by relying on the CLT - simply because the total voltage (current) is the sum of all single cell voltages (currents).

Random PWM methods based on probabilistic distributions of the switching intervals are well known in literature since long time [9]-[11] and were also applied to multilevel converters 

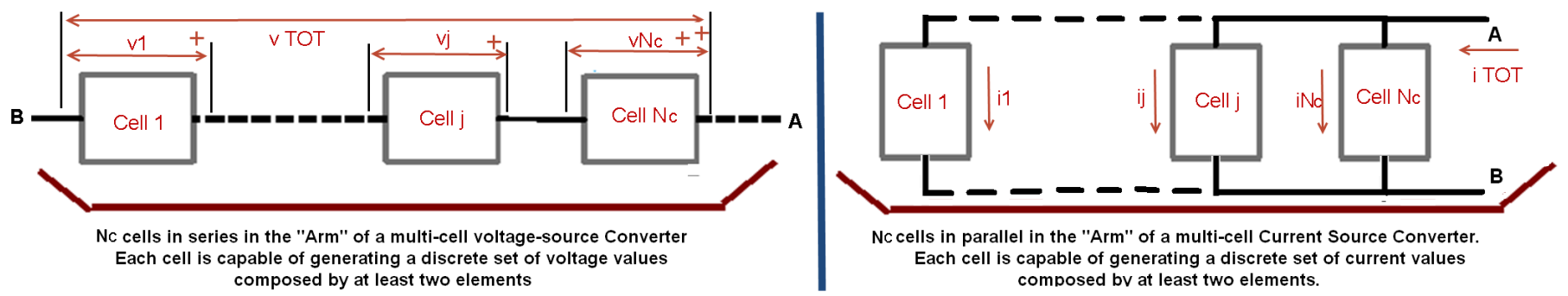

Figure 1: The structure of a voltage-source (left) or current-source (right) multi-cell "arm" in which the total voltage $v_{T O T}$, or the total current $i_{T O T}$, can be ruled by the Central Limit Theorem when $N c$ is sufficiently large.

[12] in order to achieve desired performance effects, often related to exigencies of creating spread-spectrum waveforms for mitigation of EMI and/or structure-borne mechanical noise.

The proposed synthesis differs radically from those methods because it does not introduce randomness in the switching instants, but truly in the voltage (current) values generated by each cell. Each single cell indeed has the role of a discrete random variable - independent from all others - whose possible values are the voltage (current) levels that that the cell can synthesize. Owing to the CLT, the large number of cells itself assures the accuracy of the total voltage (current) across their series (parallel) connection. The paper will also describe a hybrid approach derived to achieve similar performances with reduced number of cells that would not otherwise allow the manifestation of the effects of the CLT.

All figures possess a resolution of 400 DPI. Their details are easily readable by magnifying the electronic version of the paper.

\section{The Main Principle}

The approach is inspired by a corollary of the CLT [8], the fundamental law of nature ruling stochastic phenomena whose effects manifest themselves as sum of independent random contributions. The more numerous such random contributions are - modelled as random variables - the more the distribution of their sum approximates a Gaussian ("Normal") law centered around an expectation value equal to the sum of the expectation values of the single independent variables. The pivotal aspect stated by the CLT concerns the variance of such a sum, which becomes smaller and smaller as the number of added variables becomes greater and greater, even if their probability distributions are different [8]. This concept has been simply observed, recalled and applied by the authors to the sum of the voltages of several voltage-source cells, when they are connected in series to form an "arm" of a multilevel voltage-source converter. The observation can be equivalently applied to the sum of the currents of several parallelled current-source cells composing an "arm" of a multilevel current-source converter. These two dual networks are illustrated in Fig. 1 and from now onwards only the case of voltage-source multilevel converters will be discussed, being the case of current-source ones derivable by straightforward physical and conceptual duality.

The principle is elucidated here for the simplest case constituted by the series of voltage-source two level cells, each one capable of synthesizing a voltage of both polarities, $+V d c$ or $-V d c$, with $V d c>0$. More in general, the concept is applicable to any type of cell capable of generating at least two discrete voltage (or current) levels. The Nc cells are all independent among each other, no predefined commutation pattern and no exchange of information among them exist. This peculiarity leads to less components and to a very robust switching scheme thereby increasing the system tolerance towards cell failures. Each $j^{t h}$ cell, with $j \in\{1 . . N c\}$, possesses an internal uniform independent (pseudo)random generator $R_{j}$ in $[0,1]$ and it receives, from an external agent (e.g. the central converter controller), just the "reference probability" $p(t)$ for the value $+V d c . p(t)$ is broadcasted identical to all cells. The denomination means that $p(t)$ is the probability of the event: "Output voltage $=+V d c$ at the instant $t$ ", which the external agent desires to be identical for all independent cells. The reader already notices a significant simplification brought by the concept because the external agent needs to broadcast just one analog command signal to all cells belonging to a specific "arm", a feature that, for example, can be easily achieved by a dedicated, encrypted and interference-resistant radio link for each "arm". The synthesis of the cell voltages occur at discrete time instants separated by the interval $T s>0$, not necessarily constant. Independently inside each single $j^{\text {th }}$ cell, and independently for each specific discrete time instant $t^{*}$, the value of the random generator $R_{j}\left(t^{*}\right)$ at $t^{*}$ is observed and compared with the received probability $p\left(t^{*}\right)$ at $t^{*}$. Finally, the voltage generated by the cell at its output terminals - and kept constant during the whole time interval $\left[t^{*}, t^{*}+T s\right]$ - is chosen according to Eq.1a

$$
\begin{gathered}
\left\{\begin{array}{ccc}
+V d c & \text { if } & R j\left(t^{*}\right)<p\left(t^{*}\right) \\
-V d c & \text { if } & R j\left(t^{*}\right)>p\left(t^{*}\right)
\end{array}\right. \\
E\left[v_{j}\left(t^{*}\right)\right]=\left(2 \cdot p\left(t^{*}\right)-1\right) \cdot V d c \quad \text { b) }
\end{gathered}
$$

From this description and the chosen discrete probabilistic scheme it is immediate to infer that the $N c$ voltages generated by all $N c$ cells are discrete-time independent stochastic processes defined, at each discrete instant $t^{*}$, by the binary random variables from Eq.1a. The ensemble expectation of each process at the instant $t^{*}$ is given by Eq.1b. When the value $p\left(t^{*}\right)$ "broadcasted" to all cells by the external agent (e.g. central controller) is 1 , then all cells synthesize $+V d c$ with probability 1 and the total voltage $v_{T O T}\left(t^{*}\right)$ is the maximum that the "arm" composed by the Nc cells in series can provide. Conversely, when the value $p\left(t^{*}\right)$ is 0 all cells synthesize $-V d c$ with probability 1 and $v_{T O T}\left(t^{*}\right)$ is the minimum that the "arm" can provide. For any other value $p\left(t^{*}\right)$ strictly inside $[0,1]$ some cells synthesize $+V d c$ and some others $-V d c$ without any possibility to determine a-priori which cell does what. The switching decision inside each cell is purely local and independent from those of the other cells. Nevertheless, when Nc is sufficiently large such a "switching anarchy" at the level of the single cells does not matter for 
the ultimate quantity of interest, which is only $v_{T O T}\left(t^{*}\right)$. The nature itself indeed - acting through the model represented by the CLT - assures that the higher $N c$, the less probable it is that the random variable $v_{T O T}\left(t^{*}\right)$ deviates significantly from its expectation $\left(2 p\left(t^{*}\right)-1\right) \cdot V d c \cdot N c$, simply because the variance of $v_{T O T}\left(t^{*}\right)$ is monotonically and non-linearly decreasing with $N c$ [8].

It descends, as corollary of greatest importance, that the higher $N c$, the more probable it becomes that the ensemble average of the stochastic process $v_{T O T}(t)$ - which represents the voltage synthesized by the "arm" - remain very close to the deterministic desired reference voltage $V_{\text {ref }}(t)=(2 p(t)-$ $1) \cdot V d c \cdot N c$. Such a voltage indeed is univocally mapped by the broadcasted "reference probability" $p(t)$, which is a deterministic control signal. The $N c$-dimensional switching configuration composed by all cells in the "arm" certainly evolves fully randomly as time elapses (it is the set of $N c$ stochastic processes, each one associated to one specific cell) but, despite this randomness, the CLT assures that, when $N_{c}$ is sufficiently large, the synthesis of the sum $v_{T O T}(t)$ can be very accurate in probabilistic sense, and the more so the higher the greater $N_{c}$ is. No centralized and/or deterministic switching commands (e.g. through fiber optics) and no communication among cells are needed. Additionally, the reliable practical realizations of the present day deterministic schemes become more expensive the higher $N c$ is, whereas the cost grows much less for the proposed stochastic synthesis. This simply because it avoids the whole infrastructure otherwise needed to guarantee reliable centrally-decided switching signals, including the reconfiguration of the carrier schemes when cells fail. Here, under total randomness, no carrier scheme is present, hence no change in deterministic modulation patterns is needed. Furthermore the higher $N c$, the more robust and more insensitive to $N_{c}$ itself - as well as to $N_{c}$ variations caused by cell failures - the synthesis becomes.

The time interval Ts of repetition of the stochastic synthesis may resemble the equivalent period of any Pulse Width Modulation (PWM) scheme. Nevertheless - differently from many deterministic PWM syntheses, including several Space Vector Modulation (SVM) cases - Ts does not need to be constant at all and it can be changed dynamically during the operation of the converter, provided that a maximum value, guaranteeing a sufficient time-discretization of the reference voltage, is not exceeded. The stochastic synthesis performed at $t^{*}$ is completely independent from the other ones performed in $t^{*}-T s$ and in $t^{*}+T s$, as well as in any other discrete instant. The reference probability $p(t)$, univocally related with the desired reference voltage, can change abruptly between two consecutive discrete instants. As a consequence, significant variations in the total "arm" voltage can occur as frequently as the chosen Ts period and, in pure principle, $V_{\text {ref }}(t)$ could swing between the extreme voltage values $-V d c \cdot N c$ and $+V d c \cdot N c$ within a single Ts interval. In reality this would be rarely allowed because of physical limitations on the maximum allowed $d v / d t$, like it is often the case imposed by the lifetime of the insulating materials. The nature of the proposed method allows to synthesize any voltage waveform sampled at Ts rate, even with possibly variable Ts. Clearly when the structure of at least some cells creates non symmetrical voltage values (e.g. half-bridge cells) also the total output voltage will be consequently influenced. Variants of the approach can reduce the commutations by introducing local states at cell level [7].

\section{Suitability of the Approach For the Future Switch, Production AND System TeChNOLOGIES}

I) The "reference probability" $p(t)$ is a single scalar signal that just needs an elementary form of unidirectional communication from the centralized external agent (e.g. central controller) to all cells. A single, encrypted and interference-resistant, radio broadcasting channel assigned to each "arm" inside the converter suits the purpose.

II) Since the cells are many and one relies on a sufficient redundant number of them, for the purpose of synthesizing $v_{\text {TOT }}(t)$ it is not even necessary that the external agent is informed about the feedback signals from the switches that acknowledge the commutation. All such signals are always imperative in high power, but now they can remain confined inside each cell and can be dealt with locally according to the protection strategies at single cell level. Certainly, for purposes of diagnostics and protection of the whole converter some information must be eventually sent to the external agent. But such an information is much less, it requires much less channels and can be more delayed, than in the present cases of totally "disciplined" deterministic switching strategies. In such classic designs indeed the controller must be immediately informed about the failed switches and cells, in order to reconfigure the deterministic modulation pattern rapidly. The cost of assuring this performance increases with the number of cells. Conversely, the proposed stochastic synthesis naturally eliminates this need for a sufficiently large number of cells and the more cells exist the more robust against cell failures the scheme naturally becomes.

III) Like other well-known random PWM schemes [9]-[11] the method offers "spread spectrum" possibilities in the synthesized voltages and the consequent currents. This simplifies the ElectroMagnetic Interference (EMI) and filtering aspects already at the single cell level. The possibility to use a variable synthesis interval Ts improves this aspect further.

IV) The method is clearly suitable for "arms" composed by a large number of cells of possibly smaller ratings to realize the converters for power systems applications, like HVDC. This suits well mass production of several smaller standardized cells (cheaper by economy of scale), high design flexibility and it favours the essential fault tolerance in such applications. In turn, cells of reduced ratings suit better emerging faster semiconductor switches with minimal switching losses like $\mathrm{SiC}$ or $\mathrm{GaN}$, for which also switching frequencies and losses will no longer be a limitation as today.

V) Extensions of the presented principle can be conceived in which different, or even additional, sources of randomness are introduced in each cell. One can conceive for example variants that use the phases of the local oscillators as additional random variables, or variants that introduce probabilistic correlations among the cells or, again, variants using different distributions for the random generators. 

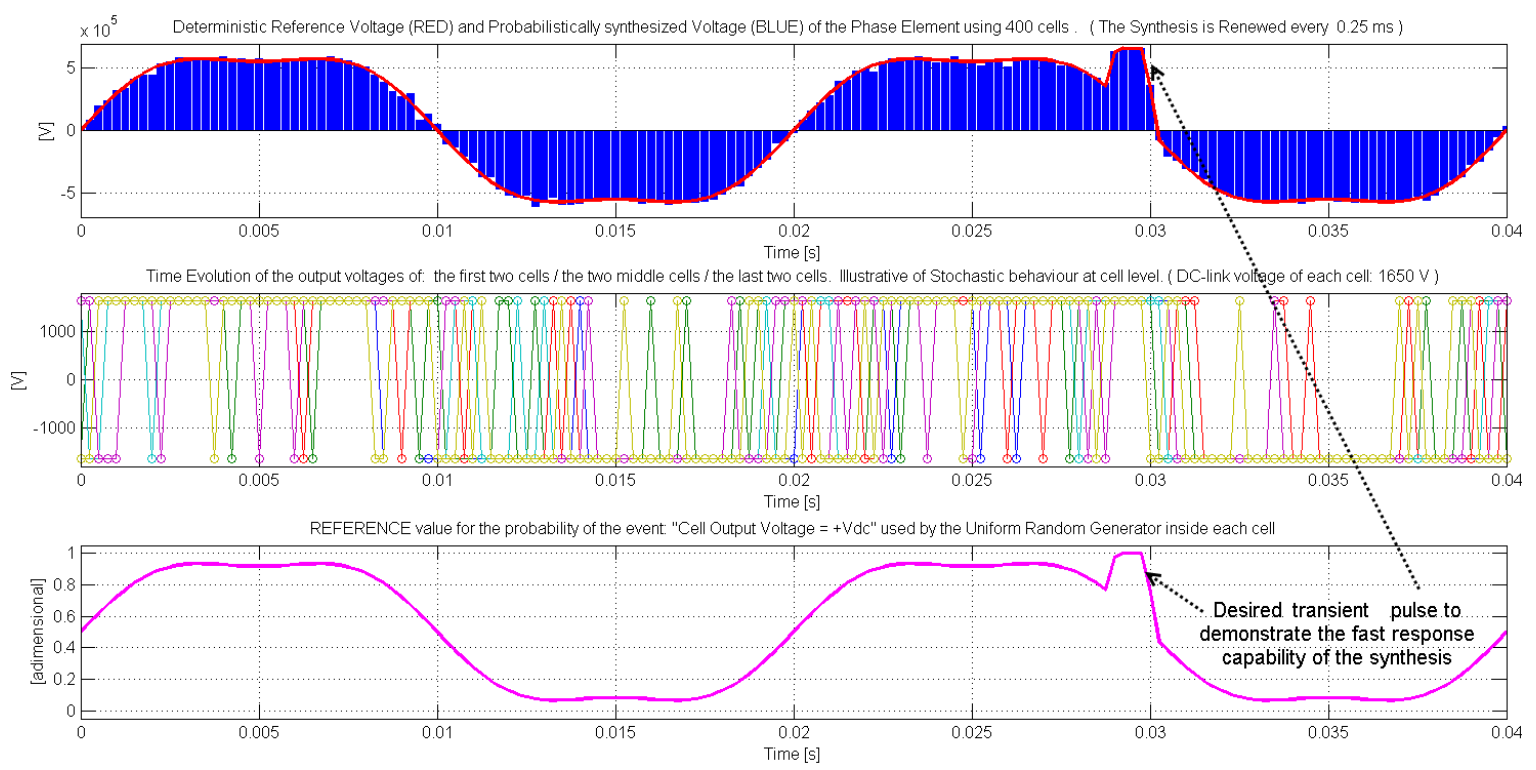

Figure 2: Simulated stochastic synthesis of a $50 \mathrm{~Hz}$ phase voltage with $1 / 6$ third harmonic injection and desired transient fast pulse, for an "arm" of a $\pm 320 \mathrm{kV}$ HVDC converter composed by 400 cells with $1650 \mathrm{~V}$ DC-link voltage, each one based on $3.3 \mathrm{kV}$ switches. Please observe the good synthesis of the transient pulse willingly added to the reference probability in order to show the fast response capability of the method.

\section{Observations ARound the Heat Generation}

Some readers might object that the method is prone to demand a greater number of switching events than what is already possible with the multilevel deterministic syntheses of today, hence it would lead to excessive conversion of energy in heat form, which is often considered wasted in several applications. This conclusion belongs to narrow visions. The aspect of "losses" indeed must be evaluated over a much broader time and technological horizon in a truly system context, way more than what characterizes today's common applications. It should be recalled indeed that the undesired, yet unavoidable, conversion of energy into heat is ultimately an aspect related to the second principle of thermodynamics and to material technologies. Since ever the continuous development of such technologies brings to a steadily reduction of the "losses" through the years. Conversely, the problem of reaching a satisfactory deterministic control of an increasingly complex modular systems constituted by thousands of similar entities is a much more fundamental aspects of nature. The statistical formulation of thermodynamics, and of its second principle again, tell us since decades how difficult such a pretence will become. It will become increasingly expensive to control reliably via deterministic approaches converters that evolve towards complexities more characteristic of multicellular organisms. The proposed approach has been conceived for future material technologies and highly modular converters. One must not narrow the vision to losses only.

\section{The Method Applied to a Realistic Number of CElls PER ARM IN MOdern HVDC CONVERTERS}

The stochastic synthesis has been firstly simulated for a realistic scenario of a HVDC converter based on cascadedcell structure, rated for $\pm 320 \mathrm{kV}$ HVDC link voltage and using single $3.3 \mathrm{kV}$ switches in each cell. This choice can justify a design based on average DC-link voltage for each cell around $1650 \mathrm{~V}$ with consequent $N_{c} \simeq 400$ cells. The main quantities are shown in Fig. 2 whose lowermost graph represents the "reference probability" $p(t)$ broadcasted to all cells by the central controller. The uppermost graph compares the desired reference voltage (magenta) - a scaled version of $p(t)-1 / 2$ together with the stochastic synthesis repeated every $T s=250 \mu \mathrm{s}$ (blue). The reader can observe how the willingly requested fast transitory pulse is well synthesized too, as permitted by the chosen interval Ts. The intermediate graph shows, with different colours, six realizations of the stochastic processes representing the individual voltages synthesized by six cells, selected two at both extremes and two in the middle of the arm. It is possible to observe how, as soon as the desired reference voltage becomes close to the maximum - or minimum - values deliverable by the "arm", the cells synthesize one of their two possible output voltage values with significant more likelihood than the other. This desired behaviour originates from the broadcasted "reference probability" $p(t)$ of the event "Output voltage $=+V d c$ at the instant $t$ " that is computed univocally from the reference voltage as: $p(t)=\frac{V_{r e f}(t)}{2 \cdot V d c \cdot N_{c}}+\frac{1}{2}$.

\section{Vi. Application to a Multi-stage M2LC Converter WITH REDUCED STORED ENERGY IN CAPACITORS}

The results presented in section $\mathrm{V}$ addressed the case of "arms" composed by cells with constant DC-link voltages. Conceptually, this implies cells capable of exchanging a significant amount of energy with the surrounding environment, not only through their output terminals, but also through additional sources connected to their DC-links. In turn, for operation over long time horizons such additional sources would require to exchange the significant amount of energy among themselves and/or with other centralized energy supply subsystems. In order to respect the mandatory graph of the converter, these exchanges could not use any galvanic connection different from the series, which involves the two output terminals of each cells. But the higher the HVDC voltage, the more this topological requirement becomes technologically difficult to satisfy because of the impractical insulation levels and number of barriers that would be needed, with consequent reduction of the system reliability too. The pivotal importance of mastering insulation 


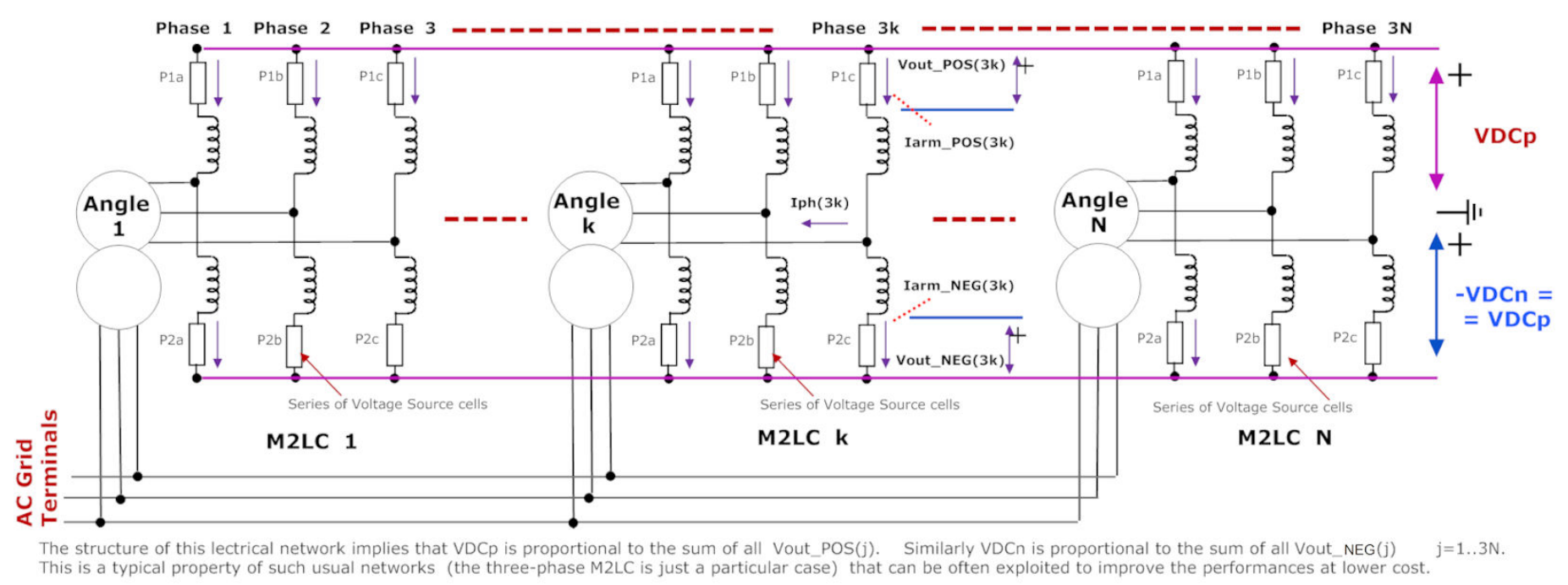

Figure 3: Multi-stage M2LC Converter capable of using high-voltage switches with reduced current rating (e.g. based on SiC technology)

technologies and materials is often insufficiently recalled in HVDC literature, which often praises the obvious modularity and scalability of the converters only. The fact that the M2LC [1]-[3] special sub-family of cascaded-cell converters eases so much the insulation endeavours around HVDC applications is one of the main reasons (not the only one) of its success, even more important than its modularity and scalability. The converters belonging to the M2LC sub-family do not need to exchange significant energy across high insulating barriers, as it would be the case for cells in series, each one having the DC-link fed through a dedicated transformer winding. This latter well known multilevel structure indeed would allow, in principle, much broader operation than the M2LC sub-family, but it is technologically very unsuitable for the voltage levels characterizing HVDC applications. Obviously the necessary "balance" of the state variables in the M2LC converters must be provided by the stochastic synthesis too and, for that purpose, it is necessary to investigate which distributions of the local random generators, as well as which local (i.e. at cell level) corrective actions might be necessary to guarantee it. The present section deals with the first investigations on this research path that has begun by simulating the dynamic behaviour of the more general multi-stage M2LC structure shown in Fig. 3, where each "arm" has been operated under stochastic synthesis.
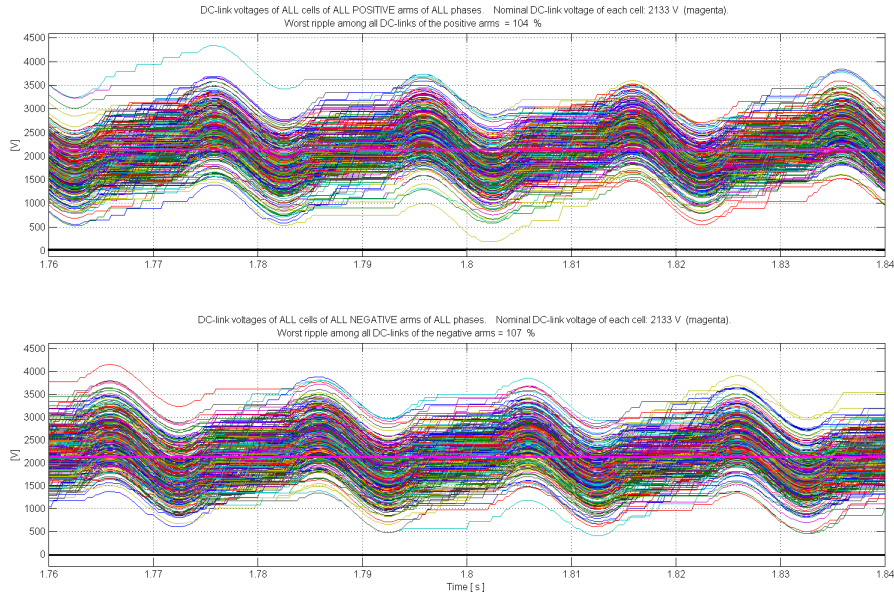

Figure 4: Capacitor voltages in the multi-stage M2LC, with only $10 \mathrm{~kJ} / \mathrm{MVA}$. They remain bounded under full stochastic synthesis
The circuit is composed by a set of $N$ three-phase M2LC converters connected in parallel at their HVDC terminals and independently fed by $N$ three-phase transformers, each one featuring a proper phase shift. The insulation technology of the transformers is not required to withstand DC components of the Electric Field, thereby reducing their costs and broadening the choice of their suppliers. The structure possesses a total of $6 \cdot N$ "arms" where each one of their total $6 \cdot N \cdot N c$ cells does not exchange energy with separated external sources. For the preliminary investigations a $1 G W$, $\pm 320 \mathrm{kV}$, multistage M2LC composed by 12 phases in $N=4$ groups has been chosen. Such a structure implies 24 "arms" in total and one phase every $30^{\circ}$. Each "arm" is conceived around lowcurrent $4.5 \mathrm{kV}$ switches (e.g. at reach of the present day $\mathrm{SiC}$ technology) with $2133 \mathrm{~V}$ nominal DC-link voltage. This design choice implies $N c=300$ cells composing each "arm" and consequently 7200 cells present in the whole converter, which would be very expensive to control deterministically with high reliability. The overall specific energy stored in the capacitors, and conventionally referred to the unit of rated converter power, is chosen to be just $10 \mathrm{~kJ} / \mathrm{MVA}$, which is a significantly lower value than the typical 30-40 kJ/MVA for a single M2LC converter [4]. Figures. 4 and 5 show selected results of the stochastic synthesis with $T s=250 \mu \mathrm{s}$ applied

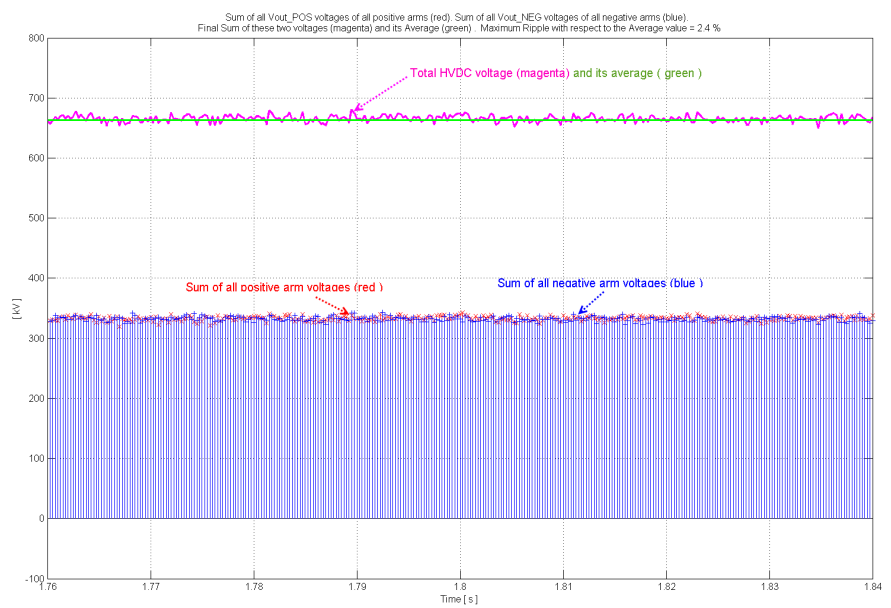

Figure 5: Total HVDC voltage in the multi-stage M2LC, with only $10 \mathrm{~kJ} / \mathrm{MVA}$ stored energy. Stochastic synthesis for each "arm". 
to this circuit. One observes that the maximum ripple on the differential (HVDC) voltage remains below $2.5 \%$ and the maximum ripple among all capacitor is naturally high by willful choice of reduced stored energy.

The theory of stochastic processes rigorously demands that the frequency decomposition be associated to each single realization only, it is not a property of the process as a whole. Nevertheless, one often observes that in some stochastic phenomena the same frequencies (in continuous spectra, for the realizations are not periodic functions in general) occur in most realizations of the process. This observation often leads to a common - yet not rigorous - empirical definition of frequency components also for the stochastic process as a whole, i.e. the conceptual set of all realizations. While always recalling that the introduction of frequency decomposition for a stochastic process lacks conceptual rigour, one can still observe the well known harmonics in the stochastic DC-link voltages shown by Fig. 4. Those harmonics are mainly caused by a deterministic consequence of the M2LC "arm" structure and they are more prominent in this simulated converter because the nominal energy stored inside it has been greatly reduced to just $10 \mathrm{~kJ} / \mathrm{MVA}$ by design. Such harmonics introduce great correlation among the stochastic processes represented by the capacitor voltages and they are NOT caused by the proposed stochastic synthesis of the arm voltages. For a chosen DC-link capacitance value (here $0.74 \mathrm{mF}$ ), the minimum amplitudes of such harmonics are unavoidably influenced by the variation of the total instantaneous energy present in the "arm", which is simply the integral function of the instantaneous power entering the "arm", as shaped by the desired synthesized waveforms.

Essentially, "above" the CLT - which lies at the basis of the stochastic syntheses of the "arm" voltages - the highly correlated contribution of the instantaneous powers entering each "arm" exists. This effect modifies the discrete set of random voltage values that each cell can generate at any given discrete instant $t^{*}$. Besides $p\left(t^{*}\right)$, also the varying DC-link voltages strongly affect the expectations of their cell output voltages now, therefore also the expectation of the total voltage $v_{T O T}\left(t^{*}\right)$ of each "arm" is ultimately affected too. Nevertheless, when one considers the total HVDC link voltage and the total grid currents, such an effect is very mitigated by the proper deterministic harmonic cancellations consequence of the transformer phase shifts.

\section{First EXPERIMENTAL RESUlts FOR ONE ARM}

A first experimental verification has been performed by implementing Eq. 1a and a pseudorandom uniform generator from [13] on a Xilinx Virtex-6 FPGA with chosen fundamental timebase of $1 \mu s$ and synthesis repeated every $250 \mu s$. The main and auxiliary boards composing the experiment are visible in Fig. 6. As a first experiment just one cell with constant DClink voltage was implemented under the rationale that, when a stochastic process can be assumed ergodic, just one realization of it already contains - along the time domain - all stochastic properties of the ensemble of all realizations, i.e. the true process itself. Figure 7 shows some periods of the reference phase voltage (magenta) featuring the typical 1/6 third harmonic injection and $50 \mathrm{~Hz}$ fundamental frequency, together with the biased output voltage of just one cell (blue), which manifests

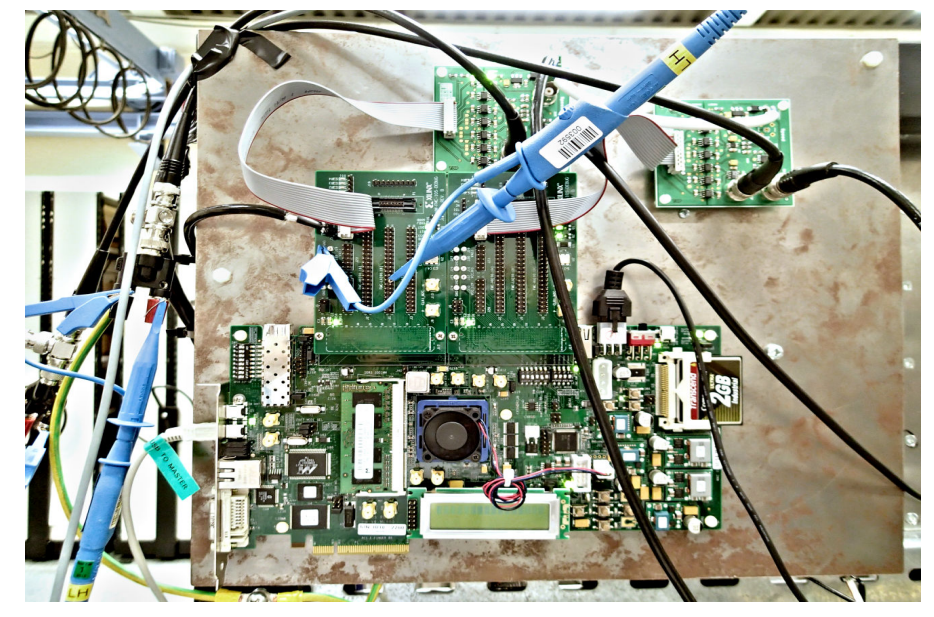

Figure 6: View of the experimental setup based on one Xilinx Virtex 6 FPGA

the effects of Eq. 1a as the rule for its random synthesis. Such a realization of the stochastic process has been recorded for $600 \mathrm{~s}$ at $1 \mu \mathrm{s}$ sampling interval and then subdivided in time windows, each one wide a single fundamental period. In order to assure the recognition of the proper beginning of the time windows in post-processing, a synchronization pulse (green) with $1 \mu \mathrm{s}$ duration has been added at each raising zero of the reference voltage. For any specific discrete time instant $t^{*}$ - univocally identified by a delay $\Delta Z\left(t^{*}\right)$ with respect to the beginning of each window - the different $N c$ values of the cell output voltage at $t^{*}$ can be added in post-processing to emulate the voltage $v_{T O T}\left(t^{*}\right)$ synthesized at the terminals of the "arm" composed by Nc cells. This technique - entirely based on the hypothesis that the stochastic process represented by the output of just one cell is ergodic - allows to explore the convergence of $v_{T O T}\left(t^{*}\right)$ to $V_{\text {ref }}\left(t^{*}\right)$ for increasing numbers $N c$ of series connected cells. This without the need to reprogram the FPGA but, mainly, it allows to explore values of NC so high that could not be implemented on a single Virtex-6 FPGA board nowadays. The results condensed in Fig. 8 were the first experimental confirmation of the method. There the reader can observe one half period of the normalized reference voltage $V_{\text {ref }}(t)$ together with different normalized sums of different realizations of the stochastic process with increasing $N c$, i.e. number of

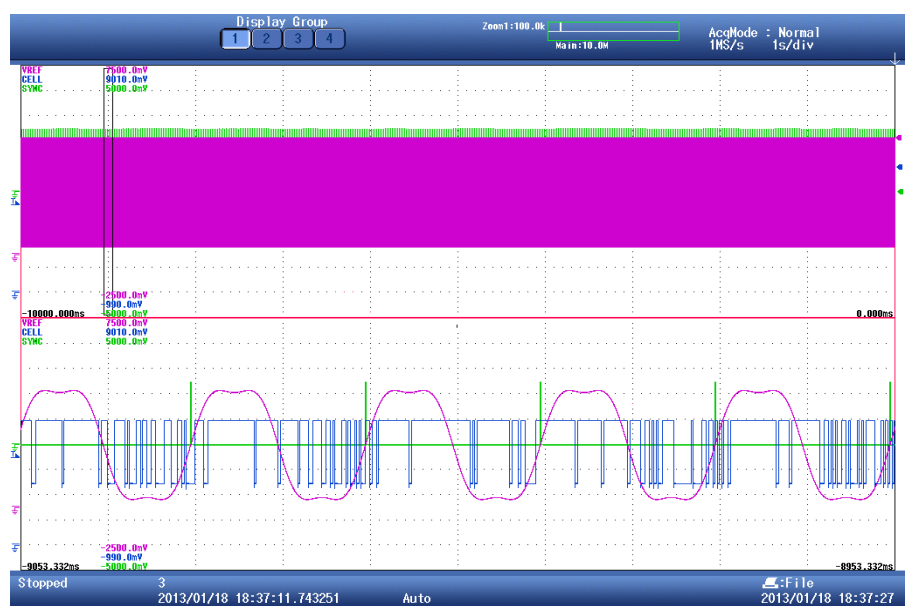

Figure 7: Reference voltage (magenta) and random switching of just one cell (blue) implemented on Xilinx Virtex-6 FPGA 


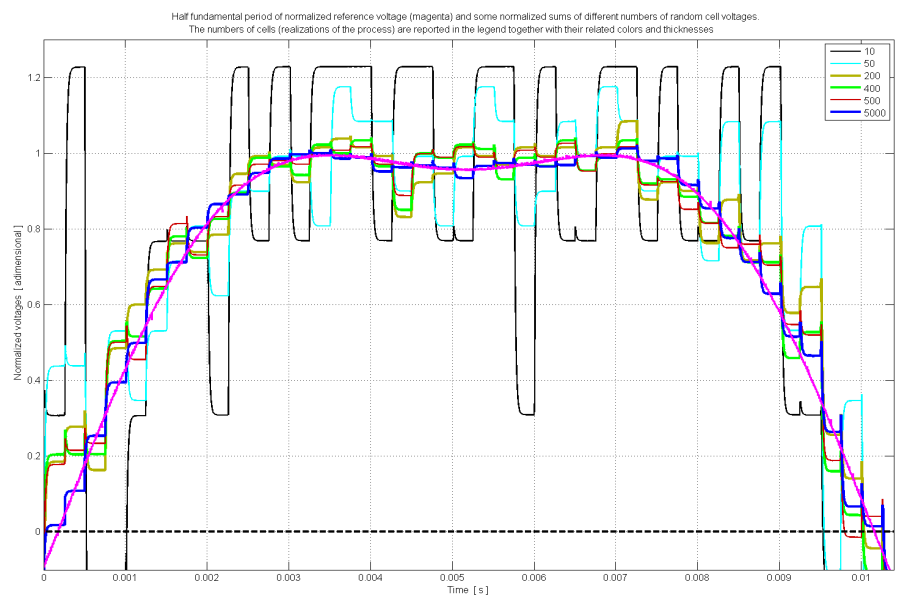

Figure 8: From the experiment: The sums of increasing numbers of cell voltages converge to the reference voltage $V_{r e f}(t)$ (magenta).

cells in series. The monotonic convergence of the normalized sums to the normalized reference voltage for increasing $N_{c}$ is confirmed, in accordance with the dictates of the CLT. After this first experimental verification, the Virtex-6 FPGA was reprogrammed to implement a complete "arm" composed by $N c=512$ cells with constant DC-link voltages. Each cell has its independent (pseudo)uniform random generator and is ruled by Eq. 1a. The output of all cells were added directly inside the FPGA, since it really emulated their series connection now. One single, properly scaled, analog output was used to measure this sum. The final result is visible in Fig. 9 and it is the most direct demonstration of the applicability of the CLT to the synthesis of waveforms in highly modular multilevel converters. Subsequently the Virtex-6 FPGA has been reprogrammed again to implement the dynamic model of one single cell with DC-link capacitor - hence no longer constant DC-link voltage - under the stochastic synthesis still represented by the simple Eq. 1a repeated every $250 \mu s$. The parameters of the cell belonging to the multi-stage M2LC structure introduced in section VI were already declared in [7] and the main ones are a sinusoidal "arm" current with $466 \mathrm{~A}$ peak, as well as $2133 \mathrm{~V}$ of nominal average DC-link voltage. Figure 10 shows the DC-link capacitor voltage emulated by the FPGA over a $30 s$ recording, firstly with a willingly unrealistic capacitance of $74 \mathrm{mF}$. Such a high capacitance has been introduced to explore whether voltage divergences, that might be caused by a fundamental problem of non-zero average power entering the arm, already appear under these idealized operating conditions. Indeed if the voltage already diverged in such conditions, with so much energy stored in the DC-link, it would mean that a fundamental conceptual problem would exist and would prevent the coexistence of the stochastic synthesis with the pivotal necessary condition represented by zero average power entering the cell. The waveform shown in Fig. 10 does not manifest divergence behaviour, which is a comforting result for the prosecution of the research. The DC-link voltage recorded, under the same operating conditions, with a more realistic capacitance of $0.74 \mathrm{mF}$ shows a significant excursion that denounces the need of a more sophisticate local control than just Eq. 1a. Proposals for this control were already mentioned and attempted in [7] and should be subject of further research on the proposed stochastic synthesis approach.

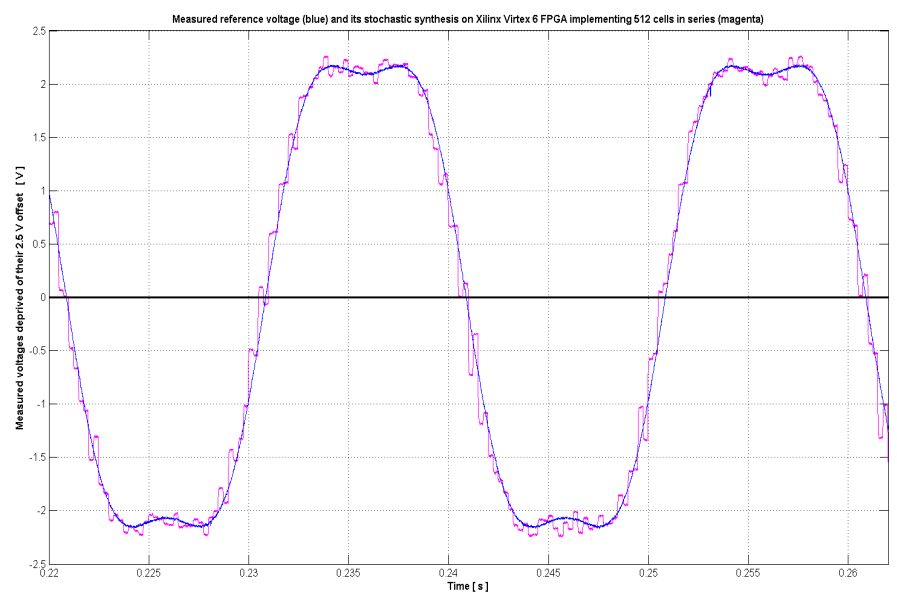

Figure 9: Stochastic synthesis for an "arm" composed by 512 cells in series. It has been fully implemented on one Xilinx Virtex 6 FPGA

\section{HyBRID SYNTHESIS FOR LIMITED NUMBERS OF Cells, hence Excessive VARIAnCE}

If the number of cells $N c$ is in the order of magnitude of few decades, well below 100, the beneficial effects of the CLT are still too limited and the variance of the total synthesized voltage remains too high for a stochastic synthesis of proper accuracy. Figure 8 proves this reality once more. The proposed approach for such cases [7] relies on a combination of the stochastic synthesis and of a local - at "arm" level - deterministic contribution that rapidly corrects, a-posteriori, the excessive error likely brought by the stochastic synthesis. The essence of the hybrid approach can be summarized as it follows by referring to Fig. 11. Additional $K<N c$ cells are added in series to the existing $N c$ fully independent ones, which remain always ruled by stochastic synthesis, as described by Eq. 1a or more elaborated variants of it. Proper system design should assure that $K<N c$ is chosen according to a function of the final desired variance of $v_{T O T}$. As previously described for sufficiently large $N c$ values, at each discrete instant $t^{*}$ the stochastic synthesis is firstly applied to Nc cells only. After the random switching events that define the voltages generated by the $N c$ cells have terminated, at the instant $t^{*}+t 1_{\text {set }}$ with $0<t 1_{\text {set }} \ll T$ s, the total voltage $v_{T O T}\left(t *+t 1_{\text {set }}\right)$ synthesized

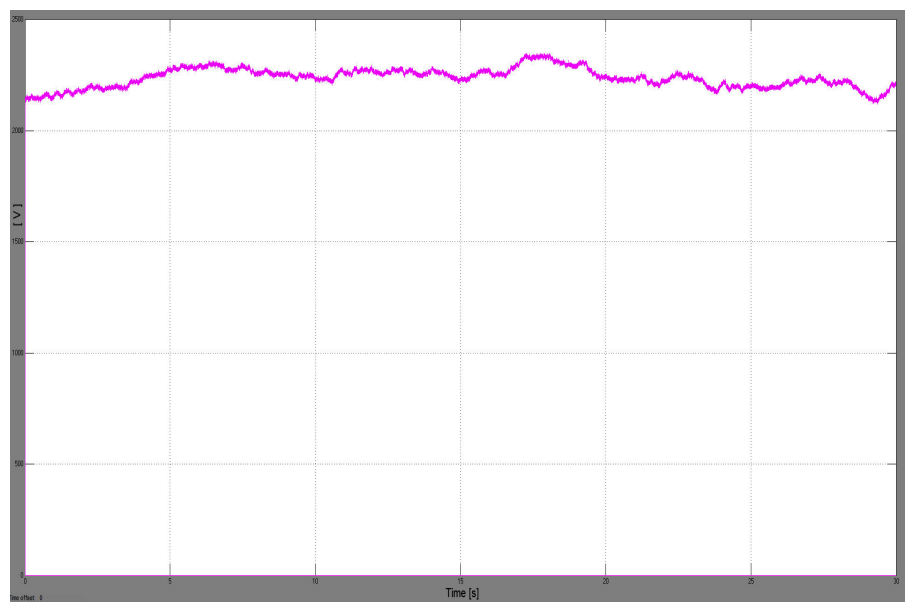

Figure 10: DC-link capacitor voltage of one cell under simplest stochastic synthesis. It does not reveal fundamental divergence 


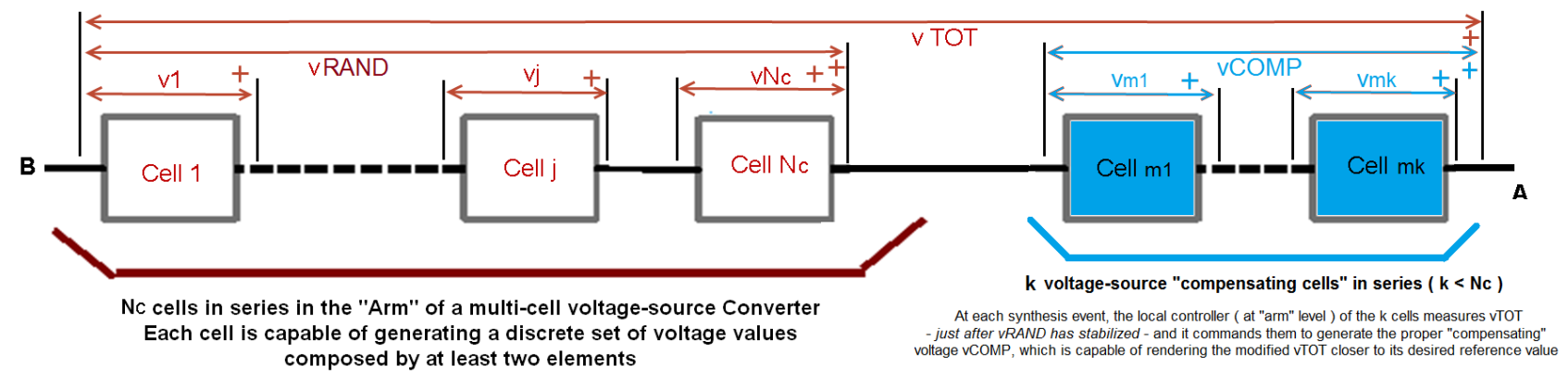

Figure 11: The principle of introducing a $K<N c$ "compensation cells" that, at any discrete-time synthesis instant $t^{*}$, correct deterministically the error between the reference value $v \operatorname{TOT}_{\text {ref }}\left(t^{*}\right)$ and the randomly synthesized value $v R A N D\left(t^{*}\right)$.

between the two extreme "arm" terminals is measured and compared with the desired reference value $V_{\text {ref }}(t *)$, which is always associated to the reference probability $p\left(t^{*}\right)$ broadcasted to all Nc independent cells, as described in the previous sections. The difference $V_{\text {ref }}(t *)-v_{T O T}\left(t *+t 1_{\text {set }}\right)$ is then compensated incrementally by the $K$ additional cells, starting to employ the cell $N c+1$ for this and proceeding further with the other $K-1$ ones, until the variance of $v_{T O T}$ is reduced to an acceptable value for the most of the interval $[t *, t *+T s]$. More in detail, after the instant $t *+t 1_{\text {set }}$ the controller ruling the $K$ cells - at local "arm" level only - commands the $(N c+1)^{\text {th }}$ cell to synthesize the most proper voltage within its capabilities to reduce the difference $V_{\text {ref }}(t *)-v_{T O T}\left(t *+t 1_{\text {set }}\right)$. After the $(N c+1)^{t h}$ cell has defined its output a new measurement at $t^{*}+t 1_{\text {set }}+t 2_{\text {set }}$ with $0<t 2_{\text {set }} \ll T$ s determines again whether the new voltage error $V_{\text {ref }}(t *)-v_{\text {TOT }}\left(t *+t 1_{\text {set }}+t 2_{\text {set }}\right)$ is now acceptable in probabilistic sense or additional correction is further needed. If so, then the cell $N c+2$ is properly commanded too and the resulting error measured again at the subsequent instant $t^{*}+t 1_{\text {set }}+t 2_{\text {set }}+t 3_{\text {set }}$ with $0<t 3_{\text {set }} \ll$ Ts. The process is repeated - for a maximum of $K$ iterations until the error voltage becomes acceptable in probabilistic sense for the major portion of the duration $T s$ of the synthesis interval. Proper design should assure that $K$ is conveniently smaller than $N c$ and that all elements in the set $\left\{t 1_{\text {set }} . . t K_{\text {set }}\right\}$ add to a value acceptably smaller than Ts. This algorithm is still a carrierless and very simple iterative scheme, based on successive approximations, that can implemented on a "local arm controller" which commands the $K$ additional "compensating cells" only.

\section{CONCLusions}

The paper has presented, and given first experimental verification of, an unconventional stochastic approach to synthesize voltage (or currents) in "arms" of highly modular voltagesource (or current-source) multi-cell multilevel converters for high-power HVDC and FACTS applications. The approach is carrierless, it eliminates the critical structuralism in the switching patterns as well as several centralized command lines to the switches, thereby leading to greater system fault tolerance and cost reduction. The higher the number of cells the more the synthesis becomes robust and accurate as natural consequence of the CLT. This is a key difference when compared to the typical deterministic carrier-based syntheses whose implementation complexity will unavoidably increase with greater system modularity. Such is the simple natural consequence of pretending to rule deterministically any large complex system, like the multilevel converters are envisaged to become, even more than today. Nature teaches us - e.g. via the statistical thermodynamics model - that ultimately such systems must be ruled stochastically, not deterministically. The paper has presented the first results of applying the method to multi-stage converters of M2LC structure with cells deprived of separated energy supplies. The first experimental results are encouraging also in this case; they show that the capacitor voltages do not diverge under random operation of the cells. Yet much more research is certainly needed. The paper has also introduced a hybrid variant of the method, which has been specifically conceived for the cases when a too limited number of cells cannot guarantee sufficiently reduced variance in the syntheses via the CLT only.

\section{REFERENCES}

[1] R. Marquardt, "Modular multilevel converter: An universal concept for HVDC-Networks and extended DC-Bus-applications," in Power Electronics Conference (IPEC), 2010 International, 2010, pp. 502-507.

[2] M. Glinka and R. Marquardt, "A new AC/AC multilevel converter family," Industrial Electronics, IEEE Transactions on, vol. 52, no. 3, pp. 662-669, 2005.

[3] S. Allebrod, R. Hamerski, and R. Marquardt, "New transformerless, scalable modular multilevel converters for HVDC-transmission," in Power Electronics Specialists Conference, 2008. PESC 2008. IEEE, 2008, p. 174179.

[4] B. Jacobson, P. Kalsson, G. Asplund, L. Harnefors, and T. Jonsson, "VSCHVDC transmission with cascaded two-level converters," in Proceedings CIGRE 2010 (paper B4-110), 2010, ABB HVDC.

[5] N. Kirby, N. M. Macleod, D. Stidham, and M. Reynold, "Tres amigas : A flexible gateway for renewable energy exchange between the three asynchronous AC networks in the USA," in CIGRE 2012.

[6] P. Tenca, "Theoretical and practical aspects characterizing the research on multilevel converters," in ECPE Workshop on Advanced Multilevel Converter Systems. V Vsters, Sweden, 2010 Sept. 28-29: European Center for Power Electronics (ECPE).

[7] P. Tenca, "Stochastic waveform synthesis for multilevel converters via the central limit theorem of the theory of probability," $A B B$ Corporate Research in "Research Disclosure", vol. February 2013, no. Database Number: 586020, p. 15, 2013. [Online]. Available: http://www.researchdisclosure.com

[8] B. V. Gnedenko, The theory of probability. Chelsea, 1966.

[9] J. T. Boys, "Theoretical spectra for narrow-band random PWM waveforms," Electric Power Applications, IEE Proceedings B, vol. 140, no. 6, p. $393400,1993$.

[10] A. Stankovic, G. Verghese, and D. Perreault, "Analysis and synthesis of randomized modulation schemes for power converters," Power Electronics, IEEE Transactions on, vol. 10, no. 6, pp. 680-693, Nov. 1995.

[11] A. Stankovic, G. Verghese, and D. Perreault, "Randomized modulation of power converters via markov chains," Control Systems Technology, IEEE Transactions on, vol. 5, no. 1, pp. 61-73, Jan. 1996.

[12] D. S. George and M. R. Baiju, "Space vector based random pulse width modulation scheme for a 3-level inverter in open-end winding induction motor configuration," in Industrial Electronics (ISIE), 2012 IEEE International Symposium on, 2012, p. 742747. [Online]. Available: http://ieeexplore.ieee.org/xpls/abs_all.jsp?arnumber=6237181

[13] G. Marsaglia, "Yet another RNG," Posted to the electronic billboard sci. stat. math, August, vol. 1, 1994. 